\title{
Impact of daily protein and energy intake and distribution on muscle mass and strength in Danish older individuals-The CALM study
}

\author{
Yusuke Nishimura $^{1}$, Grith Hojfeldt ${ }^{2}$, Simon Rønnow Schacht ${ }^{3}$, Kenneth Mertz $^{2}$, \\ Morten Hjulmand ${ }^{2}$, Søren Reitelseder ${ }^{2,4}$, Mads Vendelbo Lind ${ }^{3}$, Inge Tetens ${ }^{3}$ and Lars Holm ${ }^{2,1}$ \\ ${ }^{1}$ School of Sport, Exercise and Rehabilitation Sciences, University of Birmingham, Birmingham, United Kingdom, \\ ${ }^{2}$ Institute of Sports Medicine, Department of Orthopaedic Surgery M, Bispebjerg Hospital, Copenhagen, Denmark, \\ ${ }^{3}$ Vitality - Centre for Good Older Lives, Department of Nutrition, Exercise and Sports, University of Copenhagen, \\ Copenhagen, Denmark and \\ ${ }^{4}$ Department of Biomedical Sciences, Faculty of Health and Medical Sciences, University of Copenhagen, Copenhagen, \\ Denmark
}

\begin{abstract}
The importance of dietary protein for the maintenance of muscle mass and strength is heavily discussed. However, adequate energy intake is an underlying assumption but often not considered. In this study, we investigated the impact of daily intake and meal distribution of both protein and energy on muscle mass and strength. In a cross-sectional study, a total of 184 older individuals (gender: 86F/98M, age: $70.2 \pm 3.9$ yrs, BMI: $25.4 \pm 3.7 \mathrm{~kg} / \mathrm{m}^{2}$; means $\pm \mathrm{SD}$ ) were recruited. Participants underwent a 3-day weighed dietary record, Dual-energy X-ray Absorptiometry (DXA) scan, hand-grip strength, and Maximal Voluntary Isometric knee-extension Contraction (MVIC). Participants were divided into two categories: lower (LOW; $<0.83 \mathrm{~g} /$ adjusted(a)BW/day) or higher $(\mathrm{HIGH} ; \geq 1.1 \mathrm{~g} / \mathrm{aBW} /$ day $)$ protein intake levels analysed by gender to characterize a daily protein and energy intake pattern. Main meal protein and energy intake distributions were calculated, and correlations were made. Further, energy intake at breakfast and lunch divided by total energy intake (energy ratio) was correlated with appendicular skeletal muscle index (ASMI), hand-grip strength, and MVIC were determined using the LOW/HIGH-protein-intake categorization. Further, gender-specific ASMI, hand-grip strength and knee extension were compared based on the following four distinct daily protein intake ranges: $<0.66 ; \geq 0.66-<0.83 ; \geq 0.83-<1.1 ; \geq 1.1 \mathrm{~g} / \mathrm{aBW} /$ day. A positive correlation appeared between protein and energy intake in all main meals $(r \geq 0.57, \mathrm{p}<0.0001)$. In the LOW category, positive correlations were found between energy ratio and ASMI $(r=0.16, p=0.048)$, hand-grip strength $(r=0.40, p=0.0009)$, and MVIC $(r=0.36, p=0.0019)$, whereas no associations were found in the HIGH category. ASMI, hand grip, and MVIC were similar regardless of the protein intake ranges, though with women being lower than men $(\mathrm{p}<0.05)$ in all outcomes. These results show that total daily protein intake did not affect muscle mass and strength in our cohort. However, our data demonstrate that greater energy intake in breakfast and lunch relative to total energy intake is associated with higher muscle mass and strength, particularly when protein intake is lower than $0.83 \mathrm{~g} / \mathrm{aBW} /$ day, indicating the potential importance of meal energy content at lower intakes of protein.
\end{abstract}

\section{Conflict of Interest}

There is no conflict of interest. 